\section{Ultrasound Identification of Patella Fracture}

\section{To the Editor:}

The use of ultrasound as an adjunct technology is being increasingly employed for the diagnosis and treatment of musculoskeletal injury. Applications for its use include identification of radiolucent foreign bodies, fluid collections, and bony fractures. Advantages of sonography include portability, lack of ionizing radiation, and the ability to obtain multiple images in a relatively short period. We describe its use in the identification of a transverse fracture of the patella and compare it to a standard radiograph. This application of sonography has potential use in austere environments, in which access to traditional radiography is limited.

\section{Case report}

A 59-year-old male presented to the emergency department 1 day after a fall directly onto his right knee. He reported pain to the right knee with swelling and decreased range of motion. On exam, the patient was in mild discomfort, with tenderness to palpation of the right patella. There was a significant amount of soft tissue swelling, a moderate suprapatellar effusion, and decreased right leg extension. Given anticipated delays in obtaining plain radiography as a result of high patient volume in the emergency department, an ultrasound of the right patella was performed using a 10-5 MHz linear transducer (SonoSite MicroMaxx, Bothell, WA). Longitudinal images demonstrated a cortical disruption with associated hematoma (Figure 1). Radiographs confirmed

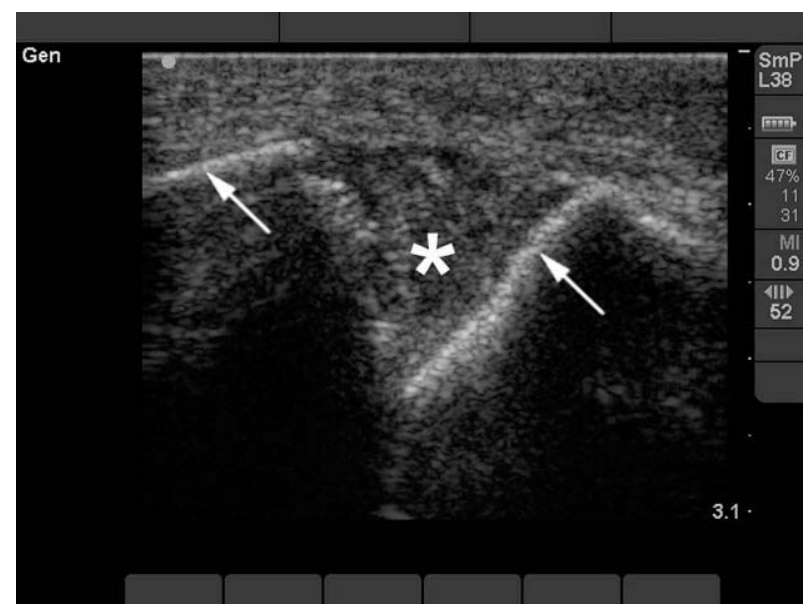

Figure 1. Longitudinal ultrasound of patella showing cortical disruption (arrows - cortex) and associated hematoma (asterisk).

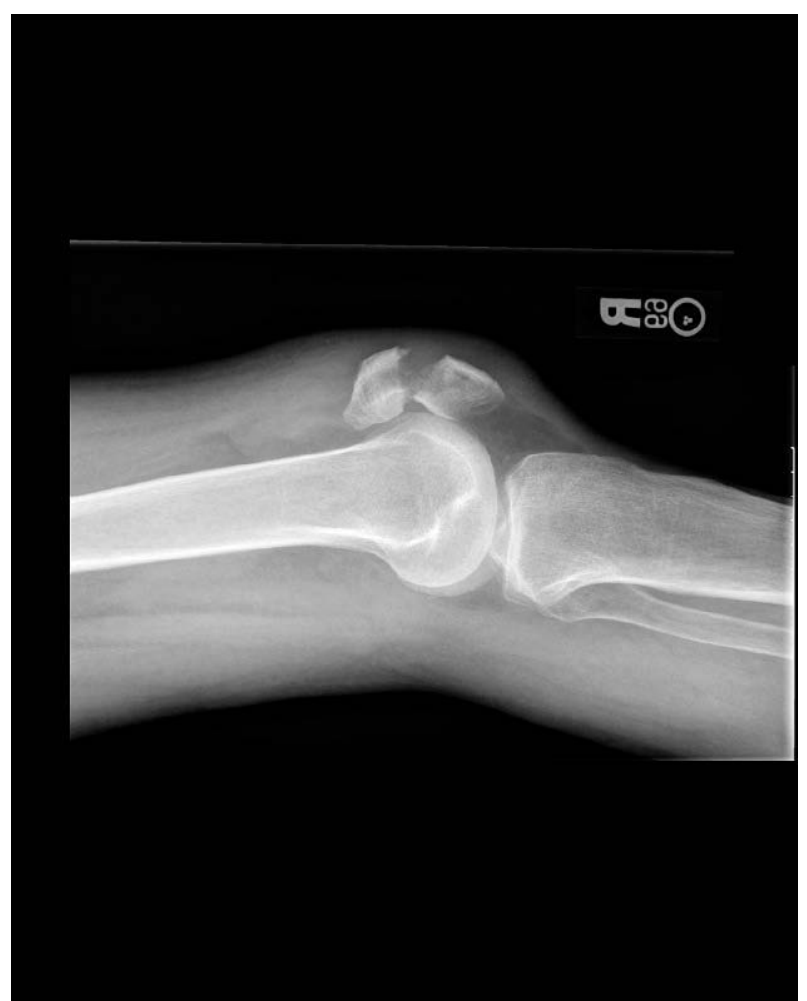

Figure 2. Lateral knee radiograph with mid-patellar transverse fracture.

a mid-patellar transverse fracture (Figure 2) with a contour identical to that obtained by bedside sonography.

\section{Discussion}

The patella is the largest sesamoid bone in the body. ${ }^{1}$ It serves as a protective barrier for the anterior portion of the knee and as a fulcrum for the extensor function of the quadriceps tendon on the lower extremity. Any disruption of the quadriceps tendon, patella, patellar tendon, or its insertion onto the tibial tuberosity limits full extension of the lower extremity. A bipartite patella occurs in approximately $2 \%$ of the general population. ${ }^{2}$ In this case, the patella develops from 2 separate ossification centers, and the 2 sections are connected by thick fibrous tissue. This should not be confused with an acute traumatic injury.

The use of ultrasound for the identification of fractures has been described previously. ${ }^{3,4}$ The exam is performed with a high-frequency linear transducer, as this provides maximal spatial resolution and is optimal for imaging superficial structures in great detail. The ultrasound exam may require the use of a water bath or stand-off pad (a gel- or water-filled cushion) for superficial osseous structures such as the phalanges and carpal 
bones. Normally the bone-soft-tissue interface is seen as a continuous, reflective, hyperechoic line. Acoustic shadowing is seen deep to the interface as a result of the high acoustic impedance of the bony cortex. ${ }^{5}$ Sonographic evidence of a fracture includes disruption of this hyperechoic line and may be associated with an anechoic or hypoechoic collection near the cortical break, suggestive of a hematoma. ${ }^{6}$ The diagnostic accuracy of ultrasound for fracture detection differs based on the location and severity of fracture. However, there is some evidence that ultrasound performs with similar sensitivity and specificity compared with plain radiography when used by experienced operators. ${ }^{7}$ Of note, while our patient suffered a substantial patellar fracture, the sonographic diagnosis of subtle patellar sleeve fractures has also been described by Ditchfield et al, ${ }^{8}$ Klerx-Melis and Watt, ${ }^{9}$ and Grobbelaar and Bouffard. ${ }^{10}$

In addition to fracture detection, additional knee pathology may be diagnosed using ultrasound. Specifically, ultrasound can be used to identify the quadriceps tendon (by sliding the probe cephalad from the superior pole of the patella) and the patellar tendon (by sliding the probe caudad from the inferior pole of the patella) and to assess for tendon disruption. In addition, knee effusions ${ }^{11}$ and meniscal ${ }^{12}$ injuries may be accurately detected using ultrasound.

To our knowledge, this is the first description of the use of bedside ultrasound by emergency physicians to diagnose a patellar fracture. The identification of bony injury using portable ultrasound units may be useful in circumstances in which radiographs are not possible or when the provider wishes to avoid the use of ionizing radiation. In fact, portable ultrasound units have already been used in mass casualty situations for identification and triage of trauma victims. ${ }^{13}$ The use of portable ultrasound for the detection of fractures could specifically affect the practice of wilderness medicine by determining whether splinting and/or medical evacuation are necessary following acute musculoskeletal trauma.

\section{R. Gentry Wilkerson MD Brooklyn, NY, USA}

Michael B. Stone, MD, RDMS Brooklyn, NY, USA

1. Christian SR, Anderson MB, Workman R, et al. Imaging of anterior knee pain. Clin Sports Med. 2006;25:681-702.

2. Weaver JK. Bipartite patellae as a cause of disability in the athlete. Am J Sports Med 1977;5(4):137-143.

3. Chen L, Kim Y, Moore CL. Diagnosis and guided reduction of forearm fractures in children using bedside ultrasound. Pediatr Emerg Care. 2007;23:528-531.
4. Tayal VS, Antoniazzi J, Pariyadath M, Norton HJ. Prospective use of ultrasound imaging to detect bony hand injuries in adults. J Ultrasound Med. 2007;26:1143-1148.

5. van Holsbeeck M, Introcaso J. Musculoskeletal Ultrasound: Sonography of the Dermis, Hypodermis, Periosteum and Bone. St. Louis, MO: Mosby; 2001.

6. O'Malley P, Tayal VS. Use of emergency musculoskeletal sonography in diagnosis of an open fracture of the hand. J Ultrasound Med. 2007;26:679-682.

7. Moritz JD, Berthold LD, Soenksen SF, et al. Ultrasound in diagnosis of fractures in children: unnecessary harassment or useful addition to X-ray? Ultraschall Med. 2008; 29:267-274.

8. Ditchfield A, Sampson MA, Taylor GR. Ultrasound diagnosis of sleeve fracture of the patella. Clin Rad 2000; 55:721-722.

9. Klerx-Melis F, Watt I. The mechanism and diagnosis of a sleeve fracture of the upper pole of the patella in children. Eur J Rad Extra. 2006;59:6-70.

10. Grobbelaar N, Bouffard JA. Sonography of the knee, a pictorial review. Sem Ultrasound CT MRI. 2000;21:231274.

11. Hauzeur JP, Mathy L, De Maertelaer V. Comparison between clinical evaluation and ultrasonography in detecting hydrarthrosis of the knee. J Rheumatol. 1999;26:26812683.

12. Najafi J, Bagheri S, Lahiji FA. The value of sonography with micro convex probes in diagnosing meniscal tears compared with arthroscopy. J Ultrasound Med. 2006;25: 593-597.

13. Sarkisian AE, Khondkarian RA, Amirbekian NM, et al. Sonographic screening of mass casualties for abdominal and renal injuries following the 1988 Armenian earthquake. J Trauma. 1991;31:247-250.

\section{Causes of Death From Avalanche}

\section{To the Editor:}

In 2 recent studies McIntosh et $\mathrm{al}^{1}$ and Hohlrieder et $\mathrm{al}^{2}$ have focused on the causes of death from snow avalanches, putting the pathophysiology of avalanche burial in a new light. This has been acknowledged by Martin Radwin in the Editorial for a recent issue of Wilderness and Environmental Medicine. ${ }^{3}$ We would like to add a comment related to the techniques of postmortem examination of avalanche victims and the study methodologies used in the cited publications.

We endorse the opinion that autopsy, strictly defined as a full postmortem examination of both external and internal organs, is the gold standard in establishing the cause of death. If autopsy is not conducted, then a documented full external examination and the review of any premortem clinical records, including radiology and computer imaging technologies, should be the required 